\title{
Radiations from Exploding Atoms
}

\author{
Phenomena Which Spontaneously Arise in the Breaking Up of Radioactive Atoms.
}

By Sir Ernest Rutherford, F.R.S.

IN choosing this title the spealier said that he had not foreseen that the object of his discourse might be misinterpreted, but the explosion which he proposed to discuss had no comnection with the common explosives used in military operations, but were the enormously more powerful phenomena which spontaneously arose in the break-up of radioactive atoms. It was most fortunate that the quantities available of these substances were too small to be of military value; and, indeed, it was a happy chance that the radiations from these atomic explosions, instead of serving as agents of destruction, found their sole practical utility in the beneficent treatment of disease. Everyone now knew that it was believed that the radiations in question were associated with the spontaneous transformation of one atom int another, the successive transformation of radium bein represented in Fig. 1. Associated with this process three characteristic types of radiations had been recosnized, and were known, respectively, as the $a$ rays, the $\beta$ rays, and the $\gamma$ rays. The explosion of an atom was an event of extraordinary violence, being at least 10 million times as powerful as ordinary chemical explosions when referred to equal quantities of matter.

In most of these atomic explosions an a particle was expelled. The a particle was an atom of matter, and the speed with which it was projected attained 10,000 miles per second, so that it carried away with it a very large amount of energy. There was, however, an explosion of a different kind, such as that of radium $\mathrm{E}$, represented in Fig. 1. In this no a particle was ex-

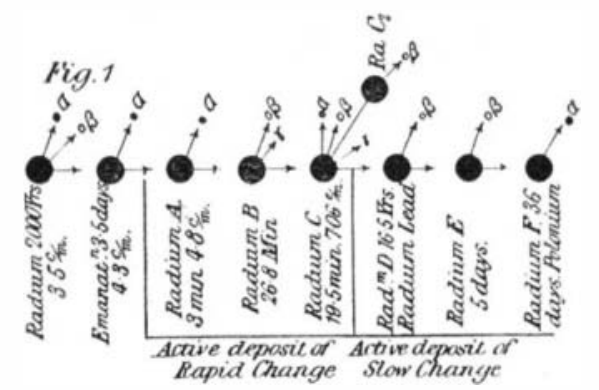

pelled, but a $\beta$ larticle merely. 'These $\beta$ particles were identical. with the cathode rays of a vacuum-tube, and were now commonly called electrons. In other cases the explosion was accompanied by the appearance of the $\gamma$ rays, which, in fact, were an almost invariable accompaniment of the expulsion of a $\beta$ particle.

An idea as to the enormous amount of energy accompanying each type of radiation could be obtained as follows: One of the simplest ways known of giving a very large amount of energy to a particle was to employ a vacuum-tube with a potential of, say, 20,000 volts between its terminals. In these conditions the atoms of the residual gases were dissociated or ionized, and these charged particles moving in the electric field attained very great speeds. There was thus a great deal of energy in the moving particles, which were, in fact, cathode rays, equivalent to the $\beta$ radiation; but there were also, as Sir J. J. Thomson had shown, positively charged particles also moving very rapidly. Now the a ray was known to be a positively charged atom of helium, but to get it moving in a vacuum tube with a velocity equal to that with which it was expelled from radioactive elements would require an electromotive force between the terminals of not 30,000 volts, or even 100,000 volts, but one of $4,000,000$ volts. The energy of expulsion of the $\beta$ particle was also very large. In the case of radium $C$, this particle was shot out with a speed corresponding to a fall through at least 2,000,000 volts, in some cases through nearly 4,$\$ 00,000$ volts. Hence the $\beta$ rays were far more penetrating than the cathode rays produced in any vacuumtube. Thus, quite apart from the interest attaching to the atomic transformation, the radiations had another interest, providing as they did examples of individual concentrations of energy of far greater intensity th:in any we could produce, or even hope to produce, in our laboratories.

The $a$ particle was an atom of helium carrying two positive charges, and it was important to measure the speed with which it moved. This could be accomplished by Sir J. J. Thomson's method of measurin the deflection the particles underwent in passing through a magnetic and through an electrostatic field. The apparatus used was represented diagrammatically in Fig.

* From Enuineering.

2. At $S$ there was a fine source of the rays, such as
wire coated with a radioactive film. The rays shot out passed between the parallel plates shown, and thence through a fine slit on to a photographic plate at $P$. If there were no field between the parallel plates,

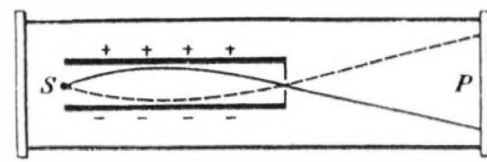

Fig. 2.

the rays struck the photographic plate centrally, but on establishing a field they were deflected up or down according to the direction of the field. Hence by rerersing the field two sets of lines were obtained on the same plate, the distance between the two constituting the measure of the deflection undergone. The result obtained when the rays were passed through a magnetic field was illustrated in Fig. 3. The elecrostatic deflection was much more difficult to measure, but it could be done, and Fig. 4 showed the result ob-

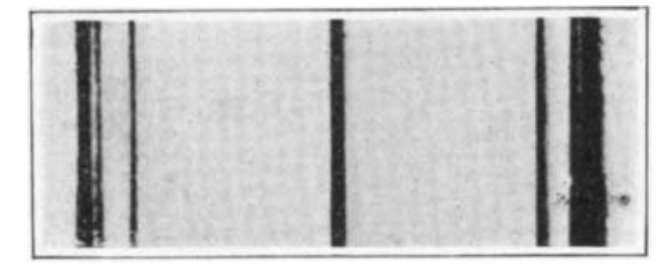

Fig. 3.

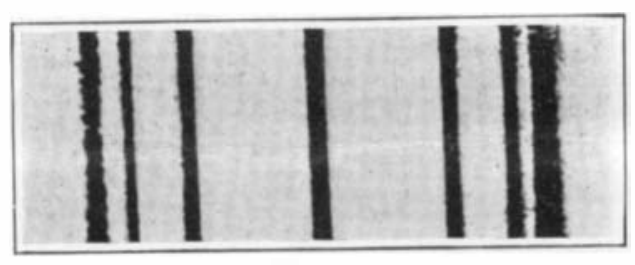

Fig. 4.

tained. It would be seen that in each photograph ther were three lines, two of which were nearly coincident in the case of the magnetic deflection. These were due to the three sets of a particles emitted by the thin emanation-tube due to the emanation, radium $A$ and radium $C$ (see Fig. 1). The three sets of particles were much more widely separated by the electrostatic deflection than by the magnetic, because while the magnetic deflection was proportional to the velocity of the particle, the electrostatic was proportional to the square of this velocity. These experiments were started in Montreal in 1902, but it was not till last year that Mr. Robinson and himself had succeeded in obtaining the photographs shown. By the aid of these photographs the speed of the a particles had been determined within a limit of error not exceeding a quarter of 1 per cent In addition the ratio $\mathrm{e} / \mathrm{m}$ of charge to mass could als be determined from the plates, and the value obtaine confirmed the view (previously arrived at) that the atomic weight of hellium was 4 , and that as an a particle it carried two charges of electricity.

The $a$ particle being expelled from the radioactive atom at a very high speed, the residue left recoiled as a gun did when fired. By similar means to those already explained, it was possible to find the velocity of thi recoil, which was sufficient to carry the atom back through some centimeters of air at 1 millimeter pressure. If the ordinary laws of mechanics held for these atomic explosions, the momentum of the recoiling mas should be equal to that of the $a$ particle expelled, and this was found by Makower to be the case.

It appeared, however, that the recoil atom carried one charge only. Since the momentum of the recoil atom was the same as that of the a particle expelled, it appeared that the ordinary principles of mechanics still held, even for these exceedingly violent atomic explosions.

As already stated, the $\beta$ rays corresponded to the ordinary cathode rays, and, like them, could be deviated in a magnetic field. Using the arrangement shown diagrammatically in Fig. 5, the rays issuing from the source at $S$ were bent round by the field and impinged on the photographic plate $P P$. On development this plate showed that in certain cases the rays after being curled round by the field were not uniformly distributed over the whole distance $P P$, but were concentrated into bands or lines, as indicated, giving a veritable $\beta$-ray spectrum which was characteristic of the particular element under investigation. Very accurate measurements had been made in this way by Rutherford and Robinson with radium $B$ and with radium $C$. Each band was due to $\beta$ particles ejected at the same speed, which could be measured from the photographs. In the case of radium $B$, the highest speed observed was 0.823 that of light, or 150,000 miles a second. Radium $C$ gave far more lines, and of these over fifty had been measured. The highest velocity attained was 0.985 that of light; and the velocity of light

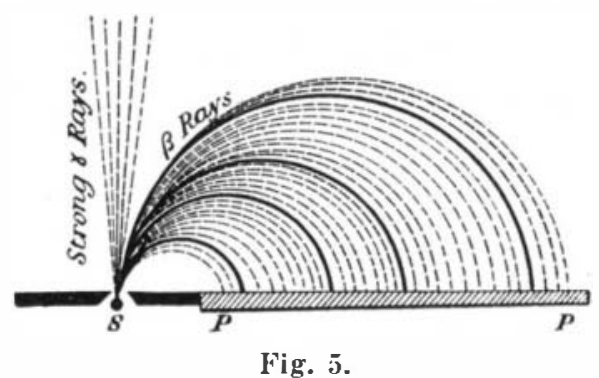

was believed to be the limiting value to which a $\beta$ particle could attain.

How was the production of these $\beta$-ray spectra to be explained? It was known, for example, that an atom of radium $C$ on an average emitted only one $\beta$ particle, yet these single particles produced, say, 50 distinct lines. The individual atom could not be responsible for these, and it was thus to be assumed that the $\beta$-ray spectrum was a statistical result due to the conjoint action of a very large number of atoms. Hence it would appear that two atoms of an element did not necessarily break up in exactly the same way. Coming next to the $\gamma$ rays, these were equivalent to $\mathrm{X}$ rays, but very much more penetrating. They to $\mathrm{X}$ rays, but very much more penetrating. They
could also be analyzed in great detail, and the results could also be analyzed in great detail, and the results
obtained were of very great interest. Some years ago obtained were of very great interest. Some years ago
Barkla had shown that when $X$ rays fell upon copper, for example, the copper, under certain conditions, gave out $\mathrm{X}$ rays which were as characteristic of copper as the $D$ line was of sodium. These characteristic radiations varied in penetrating power with the atomic weight of the radiator. It thus appeared that the $\mathrm{X}$ rays excited definite modes of vibration in the interior of the atom, and it was thus probable that the $\gamma$ rays ejected from radioactive bodies corresponded to fundamental modes of vibration in these atoms. They were thus not due to fortuitous collisions, but were as characteristic of the atom from which they came as was the fundamental note of a violin string. To test this view, the speaker and $\mathrm{Mr}$. H. Richardson had analyzed the penetrating powers of the radiation from radium $B$, and found several fundamental types. In some cases there were as many as five types, of which two might correspond to the characteristic radiations of Barkla, while the others were new types, which it would be very difficult to observe by the use of $\mathrm{X}$ rays. It thus appeared that there was nothing of an accidental character in the behavior of a radioactive atom, but that,

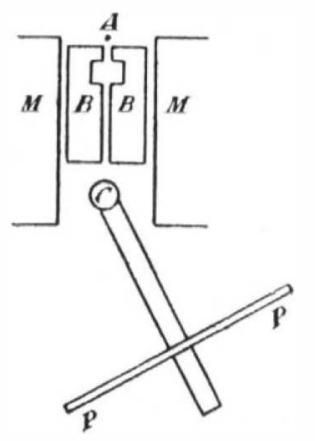

Fig. 6

in studying them, we were dealing with the modes of vibration characteristic of it, and a consideration of these should throw light on the atomic structure

Laue, Bragg, and others had actually determined the spectrum of $\mathrm{X}$ rays, but it was more difficult to accomplish the same feat in the case of $\gamma$ rays. A very long exposure, extending over a day or more, was required, and the accompanying $\beta$ radiation had to be got rid of. At Fanchester, however, Dr. Andrade and the speaker had succeeded by using the apparatus represented in Fig. 6. The source $A$ was a tube containing 
a relatively large amount of radium emanation. The radiation after passing between the two lead blocks $B B$ through a fine slit fell on, and was refiected from a face of a thin crystal of rock salt placed at $C$. A rotating arm centered on $\boldsymbol{C}$ carried a photograph plate $\boldsymbol{P} \boldsymbol{P}$, on which the radiation fell after reflection from the crystal. The crystal and lead plates were placed between the poles of a powerful electro-magnet, which deviated the $\beta$ radiation away from the plate. Without this precaution the $\beta$ rays would blacken the plate in five minutes and completely mask the $\gamma$ ray spectrum. A diagram of the $\gamma$ spectra thus obtained was shown in Fig. 7. In this case there were two lines both fairly strong, and these were found to coincide in position
(within 1 part in 300 ) with the characteristic radia-

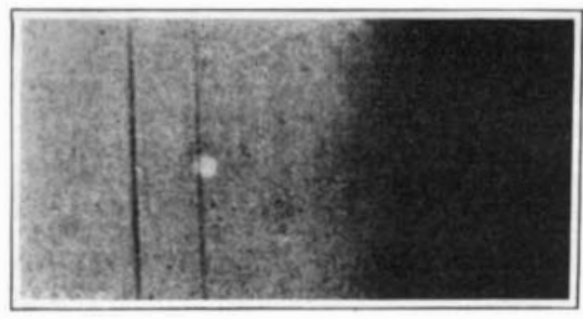

Fig. 7.

tions of lead. The plate therefore gave evidence that $R a B$ had really the same general chemical propertie as lead, although its atomic weight was 7 units more. It had proved very difficult to determine the spectra of the very penetrating $\gamma$ rays, yet success was finally attained, the wave-length found being half that of the hardest $\mathrm{X}$ rays known, having the value of less than $1 / 10$ of an Angström unit, or $1 / 50,000$ that of ordinary light.

T'he next point that arose was a difficult one, viz. the connection between the $\gamma$-ray spectrum and that of the $\beta$ rays. The one was produced by waves, the other by flying particles. In the latter case the spectrum showed that these particles were shot out with perfectly definite speeds, while the $\gamma$ ray spectrum showed that the $\gamma$ rays had equally definite frequencies. It was known that the characteristic radiation of an ele meut could be excited either by using $\mathrm{X}$ rays or cathod particles, and also that the frequency of the characteristic radiation increased with the energy of the individual cathode particles. Another result of great importance was that when $\mathrm{X}$ rays were passed through matter $\beta$ particles were ejected, the energy of the $\mathrm{X}$ ray being converted into that of the ejected particle. This being converted into that of the ejected particle. This
transfer was well shown by Fig. $s$, which was a repro-

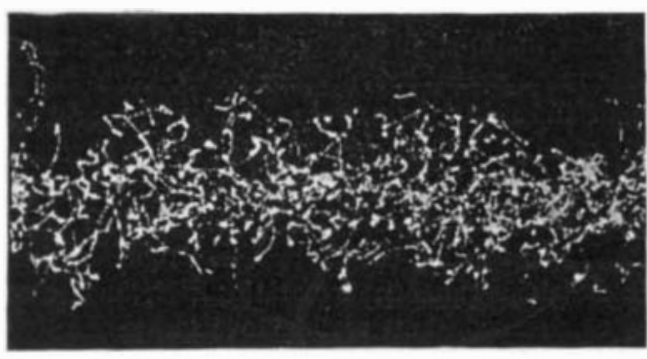

Fig. 8.

duction of a photograph by Mr. C. T. R. Wilson, obtained on passing $X$ rays through moist air. The electrons set loose ionized the gas molecules, which then erved as centers of condensation, thus showing the track of the $\beta$ particles through the air. In this case the energy of the $\mathrm{X}$ rays passed into the energy of the electrons, which in its turn was absorbed by the air. The energy of the electrons shot out was nearly proportional to the frequency of the $\mathrm{X}$ rays responsible for their ejection. The energy of the $\mathrm{X}$ rays was, moreover, given out in definite quanta depending on the frequency of the wave. This quantum of energy wa collected in the electron, and caused it to shoot out with great speed. The equality of the energy causing the emission and that of the ejected particle held approximately, but it could not yet be said that it was exact.

Marked differences in the character of the radiations were observed in different substances. Thus with radium $B$ or $C$, the $\gamma$ radiation given out was very strong, and the $\beta$-ray spectrum was banded, as already ex plained (see Fig. 5). With radium $E$, however, the $\gamma$ radiation was very weak, and in that case the $\beta$-ray spectrum had no bands, but was continuous.

There was evidently, therefore, a connection betwee the $\gamma$ and the $\beta$ rays. This had been shown more direct ly by an apparatus on similar lines to that indicated in Fig. 5. Using an $a$-ray tabe, the $\beta$-ray spectrum wa obtaine in the ordinary way. The source of radiation was then covered with a sheet of lead, and anothe exposure made. This lead stopped all the $\beta$ rays, an

only the $\gamma$ rays got through it. Nevertheless, the plate development showe exactly the same $\beta$ radiation as before, the lines or bands occupying exactly the same place as when the lead was removed. The $\gamma$ rays, in fact, which got through the lead produced new $\beta$ rays, which gave exactly the same spectrum as before. The $\beta$ rays, in these lines, therefore, would appear to be produced by the conversion of the $\gamma$ rays into $\beta$ radiation. The velocity of the $\beta$ particle produced by a wave of a given frequency was thus always the same. The energy of the $\beta$ rays was connected with the frequency of the $\gamma$ rays, and increased by definite increments, as shown in the following table:

The numerical value of Planck's quantum for the

\begin{tabular}{|c|c|c|c|}
\hline $\begin{array}{l}\text { Velocit yof } B \\
\text { Particle Expressed } \\
\text { as Fraction of the } \\
\text { Velocity of Light. }\end{array}$ & $\underset{\text { Observed }}{\text { Energy } \div 10^{23} \text { e. }}$ & $\begin{array}{c}\text { Energy } \\
\div 1.285 \times 10^{13} \\
\mathrm{e}\end{array}$ & $\begin{array}{l}\text { Nearest } \\
\text { Integral } \\
\text { Number. }\end{array}$ \\
\hline $\begin{array}{l}0.750 \\
0.868 \\
0.917 \\
0.946 \\
0.962 \\
0.969 \\
0.964 \\
0.969 \\
0.972 \\
0.978\end{array}$ & $\begin{array}{r}2.59 \\
5.16 \\
7.86 \\
10.31 \\
11.49 \\
12.82 \\
14.09 \\
15.42 \\
16.71 \\
17.98\end{array}$ & $\begin{array}{c}2.015 \\
4.010 \\
6.98 \\
8.02 \\
3.94 \\
9.97 \\
10.96 \\
12.00 \\
13.01 \\
13.97\end{array}$ & $\begin{array}{c}2 \\
4 \\
6 \\
8 \\
9 \\
10 \\
11 \\
12 \\
13 \\
14\end{array}$ \\
\hline
\end{tabular}

strong line refected from rock salt at 1 degree was $1.285 \times 10^{13} \mathrm{e}$, and referring to the table it would be seen that the energy in the ejected particle was for a number of lines practically an integral multiple of this constant. For three strong groups of low-velocity rays from radium $B$, the energies corresponded very closely with three lines in the $\gamma$-ray spectrum in Planck's law. with three lines in the $\gamma$-ray spectrum in Planck's law.
But in the case of the swifter rays, the frequency of the $\gamma$ radiation producing them was much too low, and should, in fact, if Planck's law held, in some cases be twenty times its actual value. Such a radiation would be exceedingly penetrating compared with the $\gamma$ rays. He believed, however, that these very swift $\beta$ radiations were really produced by $\gamma$ radiations of not radiations were really produce by $\gamma$ radiations of no following way.

Taking a model atom of the Bohr type, such as renresented in Fig. 9, suppose atl electron kunckenl ont

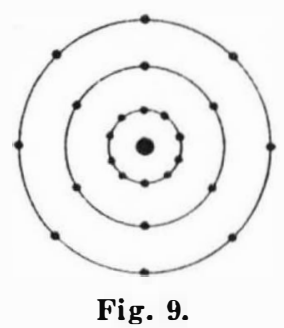

of the inner ring; then there would be a tendency for an electron to fall from the outer into the imner ring, thus setting the latter in vibration, and giving rise to a $\gamma$ radiation. In certa in cases two or three electrons might be knocked out simultaneously, and then two or three waves of the same frequency would issue together, and when these rays met another atom, the energy of the whole lot might be transferred to a single $\beta$ particle, which would accordingly be ejected at a correspondingly high speed.

As the table showed, there was a close connection between the energy of the $\beta$ particle liberated by $\gamma$ rays and the energy of these rays, and it was of interest to determine whether there was a similar connection between the energy of the $\mathbf{X}$ rays liberated when a target was bombarded by cathode rays falling through different voltages. Using a Coolidge tube, and working in conjunction with Barnes and Richardson, the

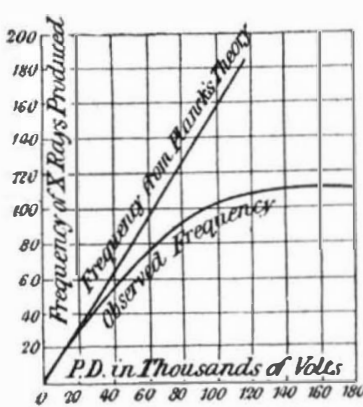

Fig. 10.

speaker had obtained the results shown in Fig. 10 The straight line showed what the connection between the frequency and the voltage should have been did Planck's law hold in this case, while the curved line showed the actual results observed. Up to a fall of 40,000 volts the two lines agreed fairly, though the discrepancy was 15 per cent; but with higher gradients the two curves diverged rapidly. The frequency reached its maximum at 145,000 volts, and underwent
110 further increase when the voltage was raised to 175,000 volts. It was clear that Planck's relation did not hold for excitation of $\mathrm{X}$ rays by electrons, except uossibly at very low speeds. It was of interest to note that the $\beta$ particles from radium $C$, which had an energy due to a fall through two million volts, did not yield $\gamma$ rays of more than twice the frequency of the $\mathrm{X}$ rays obtainable from a Coolidge tube.

A problem which remained to be solved was the maximun freffinency of the $\gamma$ rays liberated from a radioactive body. In the foregoing he had assumed that the rays came entirely from one of the inner rings of electrons, but if the nucleus itself became involved $\gamma$ rays of very much greater frequency should be produced. In fact, from theoretical considerations, he thought that $\gamma$ rays capable of passing through a mile of matter should exist, but, so far, no evidence had vet been found of this very penetrating radiation.

\section{Comets}

I HAVE lately had an interesting Fijian tradition of a bright comet, seen in $\mathrm{Fiji}^{\mathrm{j}}$ at the end of the eighteenth century, brought to my notice by Mr. Li. im Thurn "When dawn was near they saw a three-tailed star rise above the horizon. The appearance was presaged by a brightness in the sky, the comet itself soon appear ing. The middle tail was the largest, and in brightness resembled the hues of a rainbow. The other tails, one on each side, were equal to each other and white. The comet was seen for thirty-seven nights, after which our forefathers saw it no more. They took it as an omen of the death of their chief" (which, in fact, followe soon). Another account adds that a wasting sicknes decimated the people at the same time. From the approximate date given by Mr. im Thurn I conclude that the comet was probably 1799 II, in perihelion on Christmas Day, 1799, which was seen for ten days in Europe with a tail 1 degrees to 3 degrees long. I have calculated an ephemeris, and find that it approached the Warth during January, 1s00, but went into high south declination, so as to be invisille in Furope, but conspicuous to the Hijians. If my identitication is rightand I have since found that the same yroblem was sut to Mr. H. P. Hollis in 1894, and that he adopted the same comet-we are indebted to the Fijians for the knowledge that this was really a much grander comet than we should have suspected from the Kuropean accounts. We may compare the mediocre aspect of Hal ley's Comet in the British Isles with the grand spectacle that southern observers witnessed. The identification helps to fix the date of early European intercourse with Fiji and the beginning of the sandalwood trade. Mr. im 'Thurn tells me that the ship "Argo" was wrecke in Fiji about the time of the comet.

Neujmin's Comet $(a$ 1916) is another member of Jupiter's family, with a period of about 6.3 years. It was nearest to the sun on March 10th. The plane of Its orbit and direction of perihelion ale neatrly the sam as those of Encke's Comet; but this is a mere coinc dence, as the perihelion distance of Hincke is inside Mercury's orbit, that of Neujmin outside the karth's orbit; also its period is double that of Fncke. It wil be too faint for easy observation by the time this ap-

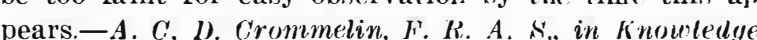

Standard Figures for Measuring Instruments INSTRUMANT-MAKbins will find some useful suggestions in a short paper by Mr. A. P. 'I'rotter in the Journal of the Institution of Electrical Engineers for 'ebruary 1st. In 1908 Mr. Trotter urepared some notes on the most suitable numerals for the scales of measur-

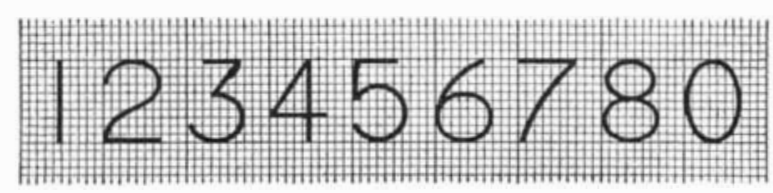

Numerals designed by Mr. A. P. Trotter, minimum thickness.

ing instruments; these were recently placeel at the disposal of the Metal Panel of the Hnineering stantards Committee, and have now been published at their suggestion. Most of the figures which Mr. 'Trutter proposes as "standard" combine the maximum of legibility and convenience with a considerable degree of elegance; his 2, 3, and 7 are admirable. A more open 5 , with the vertical and horizontal lines further to the right, may, however, be preferred; and this would enable the tails of the 6 and 9 (which it is proposed should simply be an inverted (6) to be set at a less violent and unconventional angle without danger of confusion between the 5 and 6 ; the extension of the horizontal bar of the 5 to the right, leyoud the rest of the figure, would preserve its distinction from the 3. A slightly wider + would also be au improvement.Nature. 